\title{
Promoting secondary school learners' curiosity towards science through digital public displays
}

\author{
Nuno Otero, Aris Alissandrakis, Maxmillian \\ Müller and Marcelo Milrad \\ Department of Media Technology \\ Linnaeus University \\ +46470708000
}

(nuno.otero;aris.alissandrakis;maximilian.muller; marcelo.milrad)@Inu.se

\author{
José Alberto Lencastre*, João Casal ${ }^{\star \star}$ and \\ Rui José*** \\ Centro Algoritmi \\ University of Minho, Portugal \\ +351253510307 \\ *jlencastre@ie.uminho.pt; \\ **joaocasal@dsi.uminho.pt; \\ ***rui@dsi.uminho.pt;
}

\begin{abstract}
This paper contributes to the understanding of how digital public displays can be utilized in schools taking into consideration educational goals. This work is part of a currently on-going research project that aims to promote students' curiosity in science and technology through creative film-making, collaborative editing activities, and content sharing. In order to explore the design space concerning digital public displays for schools' contexts, six workshops with secondary school teachers in two different countries were conducted to elicit sensitivities towards possible features and interaction techniques as well as inquire about expectations and technology adoption. Our findings suggest that teachers are receptive to the technology and were able to generate scenarios that take advantage of the possibilities offered by digital public displays to stimulate learning processes. However, there are several crucial elements regarding management and control of content that need to be carefully crafted/designed in order to accommodate each schools' organizational issues.
\end{abstract}

\section{Categories and Subject Descriptors}

H.5.2 [User Interfaces]: User-centered design; K.3.1 [Computers and Education]: General

\section{General Terms}

Design

\section{Keywords}

Technology-enhanced learning; participatory design; public displays; videos.

\section{INTRODUCTION}

Allowing learners to find motivating videos on important scientific topics can help learning by encouraging reflections on that content. The success of video sharing platforms has led to a growth in online video and multimedia repositories for educational purposes, $\left(\mathrm{TED}^{1}\right.$, Educational Videos ${ }^{2}$, WatchKnowLearn ${ }^{3}$, iTunes $\mathrm{U}^{4}$ ), each with tens of thousands of videos. This work is part of an EU funded research project, JuxtaLearn ${ }^{5}$, which aims at exploring these recent trends to foster learners understanding of STEM topics (Science, Technology, Engineering and Mathematics). The idea is to identify "threshold concepts" [1], concepts that constitute learning barriers, and facilitate the learners understanding through the creation and sharing of explanatory videos. These videos, together with additional data, such as quizzes, and the subsequent engagement with viewers is what we call a video performance.

The videos are assumed to be primarily aimed for the Internet, where they will be available for viewing and commenting using standard web browsers. However, we also aim to explore the distinctive role that situated displays at public locations in schools can have in JuxtaLearn video performances.

Goodyear [2] points to the fact that there is a shift in our sense of the spaces and contexts in which education takes place, as different learning activities are becoming more commonly

Permission to make digital or hard copies of all or part of this work for personal or classroom use is granted without fee provided that copies are not made or distributed for profit or commercial advantage and that copies bear this notice and the full citation on the first page. To copy otherwise, or republish, to post on servers or to redistribute to lists, requires prior specific permission and/or a fee.

MindTrek 2012, October 3-5' 2012, Tampere, FINLAND.

Copyright 2012 ACM 978-1-4503-1637-8/12/10...\$10.00.

\footnotetext{
${ }^{1}$ http://www.ted.com

${ }^{2}$ http://www.neok12.com

${ }^{3} \mathrm{http}: / /$ www.watchknowlearn.org

${ }^{4} \mathrm{http}: / /$ www.apple.com/education/itunes-u

${ }^{5}$ http://juxtalearn.org/
} 
distributed across a variety of contexts. Digital displays can provide a simple and effective way to generate shared experiences in public spaces, and when these displays are also interactive, they can be used to foster user-generated content that may enrich the video performances. Despite this potential, there are also many challenges regarding the design of the user experience around videos on the public displays and the integration into the organizational and social environment of a school. Designing digital technologies for schools needs a detailed understanding of contextual factors that will influence their adoption and actual use. In fact, some authors acknowledge that most of the innovations related to the use of ICT in schools have not impacted pedagogical or school development $[3,4,5]$. The problem is far from being trivial since online communication and interaction are not longer a separate phenomenon from children's daily lives.

In order to gain valuable knowledge regarding these complex weave of factors we have been running hands-on workshops in two European countries to engage with teachers from secondary schools. These workshops enabled us to elicit teachers' expectations towards the technology and explore possible features that fit our main purpose. The contributions of this work are twofold: a conceptual prototype of a system for video-sharing in public displays at schools, and the first steps towards the identification of a set of design guidelines for the integration of this type of system into school practices.

In the remaining of the paper we will briefly present our current conceptual framework, some initial research challenges, the methodology followed, and some preliminary results arising from the workshops.

\section{RELATED WORK}

The Dynamo display system [6], has specifically explored the use of displays in school settings. Dynamo is a large multi-user interactive display for sharing, exchanging and showing multimedia content in a communal room of a high school. Dynamo provides a GUI like interface accessible from various interaction points (wireless mice and keyboards) so that multiple users can interact with it at the same time. Dynamo allows users to connect external USB devices and access its content to display it publicly on the screen or to share it with other users by dragging it to a public area or by sending it to specific people (users had to register to be able to access some of the functionalities). During the deployment time, students used Dynamo to display and exchange photos, video and music; to create a pool of public media that anyone could use; to stage performances to the audience in the communal room; to post notices to other users; to leave content as gifts to specific people; and to engage in group discussions and interactions. Dynamo provided only the infrastructure for the content sharing. Users were ultimately responsible for creating meaningful content and appropriate the system in an adequate way for that place.

Instant Places $[7,8]$ has been deployed in a university bar and in a communal space of a secondary school. It allows users to contribute to the content that is displayed by specifying keywords on the Bluetooth names of their mobile phones. The system uses a pre-defined source for content, the Flickr photo sharing website, but allows users to contribute by specifying keywords and Flicker user IDs that the system uses to search and display photos. Apart from the fact that users cannot choose the source for the content, they have a higher degree of control over what is displayed than in a completely centrally-managed display system. Control is not as high as in a pure user-submitted display system, however, because a user is not able to display at will a specific photo. Instant Places achieves a balance between the two, leveraging on the advantages of both.

However, generating engagement is still a key challenge. Agamanolis [9] notices "Half the battle in designing an interactive situated or public display is designing how the display will invite that interaction." It is not just a matter of offering interactive features to the public. Those features must offer an obvious value and, in some cases, the display system must explicitly or implicitly invite people to interact. A study on enticement [6] has suggested specific design strategies towards encouraging people to participate. In their observations they noticed a "honey-pot effect". Because interaction with their system could only be done in a single place - the laptop provided for the effect - people would gather around that single spot creating an interest area where others would try to get close to see what the buzz was about. This increased the number of interactions with the system. They also noted that people would approach in phases: first becoming peripherally aware of the display; then focusing and socializing about it; and then actively interacting with it. To move between these phases people need encouragement to cross the thresholds, principally to active participation. Huang et al. [10] have observed that there are other ways to achieve a similar effect such as exposing others' interactions and having a dedicated core group of users.

\section{FRAMING THE RESEARCH PROBLEM}

The use of public displays at schools for the specific purpose of complementing video performances, as specified in Juxtalearn, represents a novel concept that requires further characterization and a clear framing of the respective challenges.

In JuxtaLearn, video performances are seen as an education process in which, through video creation, students can identify misunderstandings and overcome their learning difficulties. Videos themselves are the central element of that process, but they are not the main outcome. In particular, it should be clear that the video is not a finished product, but an evolving performance that should evolve with the learning process and the collaborative dialog that may emerge with the video sharing. The presentation of the videos on the public displays must be part of that process and somehow add value as an extended performance space. We thus framed the problem in terms of the unique value proposition offered by public displays, rather than as a mere problem of how to extend the web context to this medium despite the differences.

Regarding the display environment, we are addressing the public space at the school. By public space, we mean communal spaces in schools, outside the classroom context. This is aligned with Goodyear's view of how there is a shift in our sense of the spaces and contexts in which education takes place, as different learning activities are becoming more commonly distributed across a variety of contexts [2]. Using the public space of the school as a stage for learning activities, and more specifically Juxtalearn video performances, constitutes a natural extension of the process but also raises several specific challenges.

To promote engagement from people watching the videos, but also to increase the value to the overall process, video performances on the public displays should be interactive. They should allow people to engage with the performance in a way that 
is rewarding for them and that enriches the performance itself. We thus assume that students will not simply be viewers of videos but they will also be able to annotate them or in some other way engage with the respective content. This could be achieved through their mobile devices or some other interaction technology that may be available in the setting, but creating appropriate interaction mechanisms to foster interaction between users within the school/educational context and the videos is a challenge in itself. Even though interaction support has been one of the most active areas in public displays research, there are still no interaction models that are mature enough for generalized support for interaction with public displays. The challenges involved are not merely technical, as they also involve the emergence of accepted interaction practices and social rules around the shared use of displays.

The main challenge however is to design the system in a way that maximizes its value for the video performances and this requires some reflection on the intended value to be offered by public displays in the context of video performances. In particular, public displays should not be seen as simply an additional channel for presenting the videos. Extending video sharing and presentation from the web context to the public displays needs to take into a significant number of specific challenges associated with this medium, but also the new opportunities that this particular medium can create for video performances. In particular, it should be clear in what ways the use of public displays as complimentary medium may enrich the overall experience of video creation and sharing.

As a communication medium, there are two key properties of public displays that we believe may be of particular relevance for JuxtaLearn performances: their ability to create shared experiences and their ability to promote unwitting encounters with information.

\subsection{Shared experiences}

The ability to create shared experiences is clearly a distinctive element of public displays and an important part of their unique value proposition. Essentially this means that videos seen by colocated students near the display may become a common reference for conversation, discussion or comments.

This learning context is not bound to classroom rules but inserted into wider range public places. Therefore, we cannot expect it to be the setting where students will be able to reflect deeply on the content. However, it should play an important role in facilitating the transitions between "class/web" content and a physical space that is also a socialization place. A properly designed system should take advantage of the social dynamics already present in those places and make it an important part of the dynamics of the entire process, by building up interest and making videos a conversation topic.

The social visibility given to the videos presented on the public displays may also constitute an important element of reward for video creators and a motivating element for driving more students into the creation of their own performances. Seeing videos made at their school by people they know, may help driving other students to create their own videos. For a video creator, achieving to make a 'buzz' among the school colleagues may in a sense be much more rewarding than online comments from remote viewers and especially may help to create a quicker and richer feedback loop that whatever may happen within the broader and more

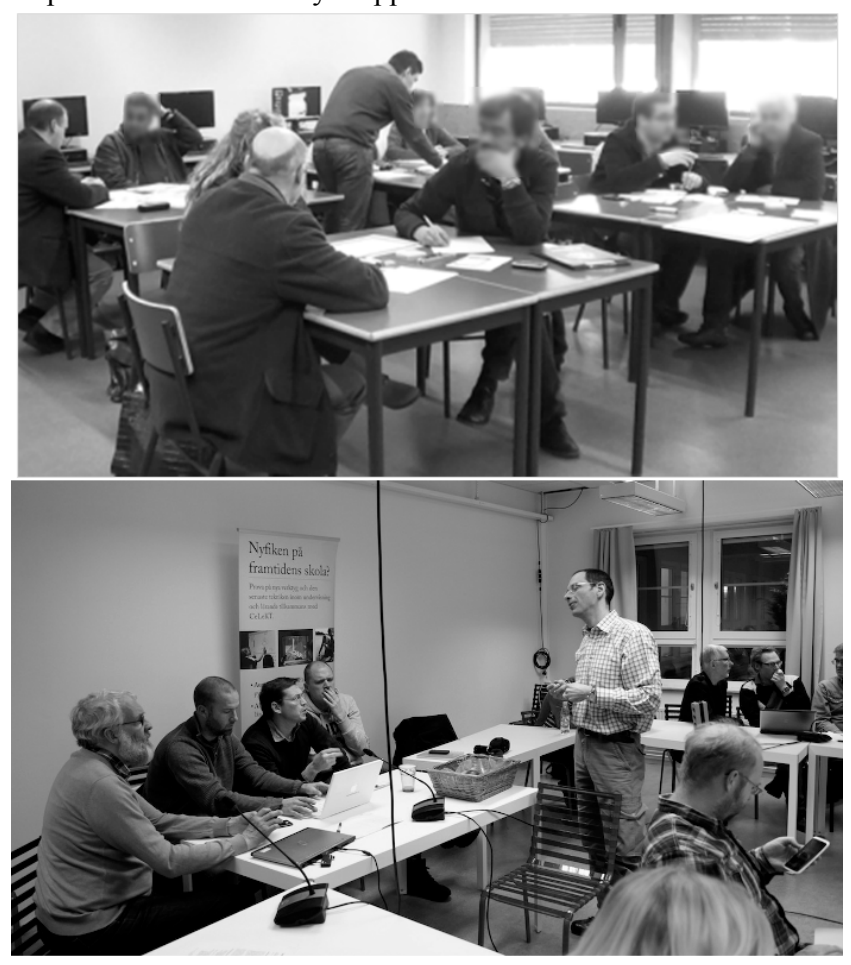

Figure 1. Photos from the workshops in Portugal (above) and Sweden (below).

anonymous context of the web.

\subsection{Serendipity}

The other key property that we want to explore is the ability of public displays to create unwitting encounters with video performances. In a time of information overload, people seek information based on multiple implicit and explicit filters that only show them what they know they need. Public displays in a communal space of a school may represent an opportunity to break away from these self-imposed content bubbles and promote a stronger awareness about other topics, activities and interests. In particular, for a school community it could play an important role in breaking traditional discipline or year boundaries and create a broader sense of community. Hopefully, these encounters should feed curiosity around new topics and science in general, as a result driving students towards topics they would not normally search for.

\subsection{Research questions}

In order to explore the design space of digital public displays for schools framed by the particular goals related to the JuxtaLearn project, we have formulated the following research questions:

- How do teachers see the potential of sharing the videos on the public displays at schools? Are they willing to promote their adoption among their colleagues?

- What particular interactive features do they think suit best the school context?

- What concerns do they think we should take into account when designing such systems? 


\section{METHOD}

\subsection{Participatory design workshops}

Our initial explorations involved a participatory design approach where we invited teachers to take part on a set of design workshops, each of which was planned to last two hours. These design workshops were intended to give us some insights into the initial questions considered but also to be able to start managing expectations and engage with the teachers in a partnership that would facilitate the deployment and testing of the prototypes in their schools. Hence, we considered important to foster ownership from the teachers regarding the design solutions to be presented.

Six workshops were run, three in Sweden, and three in Portugal (see Figure 1). In total we had the participation of 18 secondary school teachers. The data collected in the workshops include: (a) notes from the conversations taken by the researchers present at the workshop; (b) the scenarios and paper prototypes developed; and (c) the answers to the questionnaires given.

\subsubsection{Stage one: presenting the project, the initial} ideas, and fostering acceptance

In these initial workshops the goals and activities were:

a) Explain the goals of the JuxtaLearn project.

b) Explain the goals of using digital public displays to encourage further exploration of the content and facilitate the creation of groups of interest.

c) Run a hands-on activity where the teachers were invited to express their understanding of the technologies' potential and their expectations regarding the project.

d) Hand out a questionnaire that inquired about the envisioned uses of such system within the school and possible expectations regarding outcomes of its usage. More specifically the questions covered the following themes: reflect about the plausibility of the introduction of digital public displays in schools, consider the advantages and disadvantages of such technology, potential ethical issues, the use of mobile technologies and the design process underway.

\subsubsection{Stage two: exploring further the teachers' ideas about features}

For the second batch of workshops, the same groups of teachers were asked to participate. The goals and activities were:

a) Involve the teachers in further refining of their ideas regarding the digital public displays.

b) Small groups of teachers created "paper prototypes" of digital displays (see for example Figure 2).

c) Those same groups created a scenario based on the paper prototypes they developed, followed by a brief period of presentation and discussion of the prototypes and scenarios created.

d) Hand out a questionnaire that inquired about the workshop activities and possible changes regarding the envisioned uses of such a system within the school. More concretely the questions were similar to the ones proposed in the questionnaire of stage one but this time we were relying on the teachers reflections after being exposed to the presentations, discussions and creation of prototypes.

\subsubsection{Stage three: presenting the first prototype}

The final workshop, that materialized the work done on the others, had the following purposes:

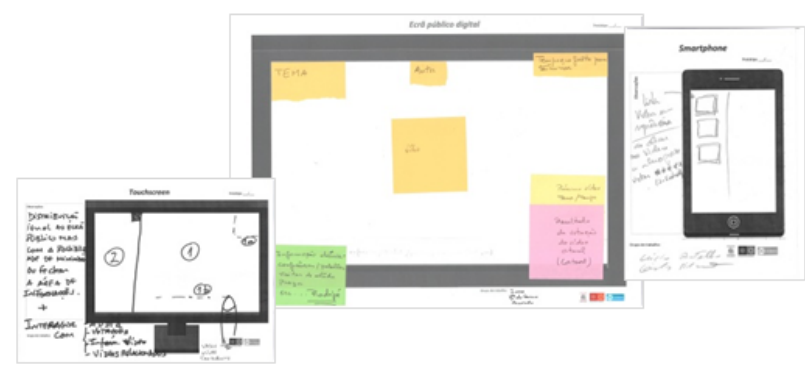

Figure 2. A paper prototype outcome from the workshops.

a) Present the first conceptual prototype.

b) Ask the teachers to, in small groups, discuss the design and features presented, and comment to what extent the prototype corresponds to their ideas (based on the work done on previous workshops).

c) In the same groups, invite the teachers to create and present a scenario based on the functionalities of the conceptual high fidelity prototype.

d) Foster a discussion about ways to initially engage students with the system and get an agreement of where the display should be located in the school.

e) Using an online questionnaire, get the teachers' individual opinions about the set of workshops. Furthermore, the questionnaire also inquired about: how teachers envision the acceptance of and contribution to the platform by the school community (colleagues and students) and what educational outcomes might arise with the use of the system.

\section{FINDINGS FROM THE FIRST TWO STAGES}

The findings reported below are based on a qualitative data analysis approach where the design team discussed together the data collected and created meaningful interpretations of it.

\subsection{Fostering curiosity}

The teachers were quite aware of the potential of the digital public displays to be used to foster the students' curiosity to particular content, for example, a specific topic to be explored further or themes of upcoming lectures. Such approach could be designed as short trailers, teaser videos, animations, or still images.

\subsection{Comment feature}

The teachers were favorable to the possibility of allowing comments to be made to the content being shown. The comments could have a dedicated section on the screen and provide functionality for e.g. direct feedback, publishing of thoughts, and part taking. However, further discussions with the teachers brought up concerns regarding free text input and input control. This puts emphasis on the need to develop control mechanisms and provide authoring functionality. 


\subsection{Quizzes}

The idea of providing and creating quizzes related to course material was mentioned in all the workshops conducted. The teachers seemed to agree that these techniques create opportunities for interaction, which might motivate the students to pay attention to the public displays, engage in activities that test their knowledge and learn about topics in a less formal way. In relation to more design-driven comments:

- Mobile phones can be used as input devices.

- Ask for multiple-choice instead of free text answers: this would additionally allow avoiding the undesired publishing of inappropriate content.

- The quizzes can be visually driven, with images and/or videos. For example "this video illustrates which law of physics" or "what would happen next".

- Allow the possibility of creating quizzes that encourage individual and group participation, and display the results as a form of quick feedback on overall performance. For example, create visualizations of (all) students' answers using a word cloud.

- Having the participation stored in the personal account of individual students could allow for the possibility to display these records on their mobile phone and track their personal improvement.

\subsection{Polls}

Some of the scenarios created suggest the use of polls within the school context. Examples mentioned by the teachers were "vote for favorite school meal", "vote for student council positions", but also "what do you want to learn about next week". According to the teachers, the polls can not only provide opportunities for educational scenarios but could also engage the students in generic school related issues.

\subsection{Displaying schools' information}

Similar to event posters, the teachers suggested the use of the public displays to communicate information about school activities, e.g. projects, group work, food menu, and sport events.

\subsection{Streaming audio to mobile devices}

According to the context or situation around an interactive digital public display and plausible scenarios in schools, the displays cannot play loud sound all the time. In fact, some designs suggested the possibility of having a display that shows several videos at once, in which case the sounds would interfere. One possible solution to counteract this constraint would be to selectively stream the audio to nearby mobile devices. By doing so, the audio could be accessible from each student's mobile. For example, a playlist could be created and maintained, changing its content automatically according to the displayed video.

\subsection{Ethical concerns around the use of interactive digital public displays}

During the workshops questions were posed to elicit teacher's opinions about potential ethical issues arising from the use of digital displays in schools. Furthermore, at the end of the workshops questionnaires were handed out and these also included some questions covering the issue. Thus, the data collected regarding ethical issues consisted of transcriptions from video recordings, notes taken by the researchers and answers to the questions present in the questionnaire.

\subsubsection{Inclusive solution to access the platform}

When researchers presented smartphones as the main interaction device an ethical question emerged: not every student has a smartphone. Furthermore, the teachers also pointed out that in some schools the use of mobile phones is highly restricted. To solve this issue, the teachers mentioned that there could be a touchscreen near the display, for the use of every student, as a second device of interaction. After reflect about this question and consulting the development team, the researchers envisioned some questions about the use of a public device to interact with the intended platform: the necessity of logging in and logging out would obstruct the use and add other issues to the interaction mechanisms (for example the improper use of an account that a student forgot to close). After presenting these questions to the teachers, the solution agreed was to avoid the need on $\log$ in on the touchscreen, reducing the features accessible on that device because some require an identified user.

Eventually, the JuxtaLearn solutions need to take into account these issues in order to facilitate the emergence of fairness in access and use.

\subsubsection{Protecting minority groups and facilitating diversity of opinions, topics and content in general} The elicitation of teacher's opinions at the workshop in Portugal pointed out the need to investigate mechanisms to protect the diversity of the schools' populations and try to promote the expression of different opinions, topics, likes and dislikes. Teachers also thought important to consider the need to safeguard the diversity of topics and not restrict the content to STEM. Such stance would allow the generation of interest to a wider audience.

\subsubsection{Reflecting different practices towards conduct}

Teachers both in Portugal and in Sweden considered important that the system to be deployed will need to allow some degree of customization in order to reflect potential differences concerning the specificities of schools' rules. This is particularly relevant for the protection of privacy.

\subsubsection{Implementing content control mechanisms in order to avoid offensive episodes}

Teachers in both countries were very sensitive to the need to find appropriate content control mechanisms. In fact, this is central to the adoption of the system since the general opinion seems to be that any incident would jeopardize the deployment of the system. Some suggestions pointed to the possibility of facilitating access only to registered users.

\section{TOWARDS A FIRST PROTOTYPE}

A conceptual prototype was built in collaboration between teachers and the development team with the researchers as mediators, has partially shown before. The teachers provided, during the participatory design workshops, the knowledge of the school context and the development team informed about the technical possibilities.

This conceptual prototype was built by means of use cases in order to be presented clearly to the teachers, and also to form a precise tool to the developers about which features have to be built and who are the users related with it. Additionally, the designs of the applications for the public display and for the interaction devices were outlined based on the paper prototypes 
built during the second workshop in Portugal and on the development team opinions. actor will be able to interact with a smartphone application and with the touchscreen. At the smartphone the student can interact with videos: vote, comment, share, see the list of the available ones, know more, answer quizzes and, obviously, push the videos to the public display. The touchscreen features available, having the restrictions presented before in mind, are see the list of videos available, know more about one and push videos to the display.

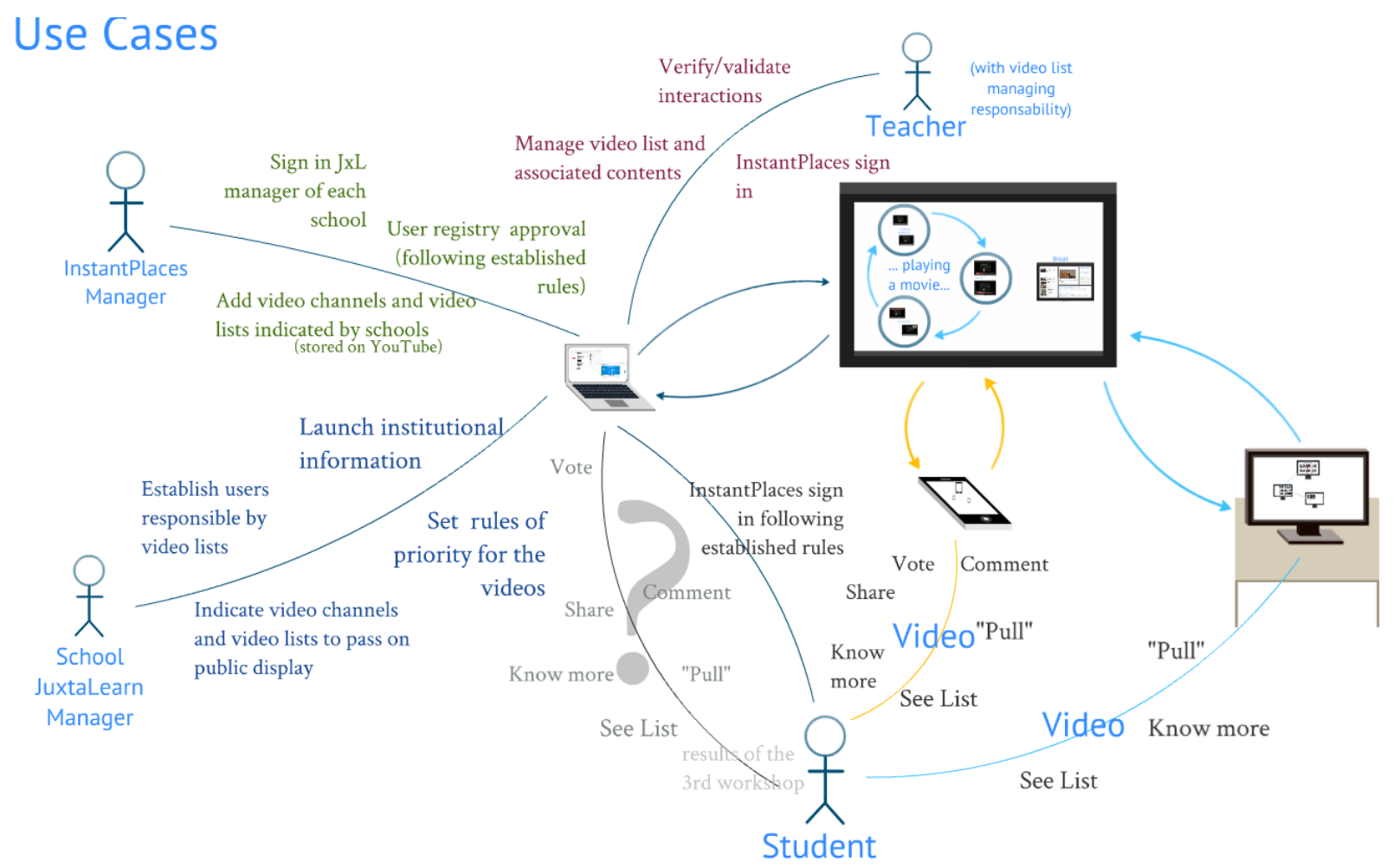

Figure 2. Use case diagram of the conceptual prototype.

The foundation for this prototype will be the InstantPlaces platform, which has the advantage of building a system upon something established. There were four actors and three interaction devices on the use case diagram presented to the participatory design participants (see Figure 3). InstantPlaces Manager, School JuxtaLearn Manager, Teacher (content manager) and Student are the envisioned actors of the platform. The planned interaction devices are the smartphone, the touchscreen and computer. Each of these has different applications and features, based on the fact that different kinds of users will interact with them. Mainly, InstantPlaces Manager will interact with a web application (computer) to assign the School JuxtaLearn Manager, with the YouTube video channels that will provide the videos, and also approve user registries following established rules. The School JuxtaLearn Manager will have a web application with features to: indicate video channels/lists to show on public display, establish users responsible for those video channels/lists, launch institutional information to the platform and set the priority rules of the video presentation. The Teacher actor (with video list managing responsibility) will also interact with a web application to manage the videos that are from his responsibility and to verify/validate interactions (like comments). Finally, the Student
Beyond the interaction features, we also presented to the teachers some design outlines of the applications based on their opinions (gathered by the mentioned methods: questionnaires, video transcriptions, notes) and on the paper prototypes built on the previous workshop.

Mainly, the teachers saw their previous labor of the workshops mirrored on this conceptual prototype. However, the secondary interaction device of the students (touchscreen) was seen as insufficient to override the ethical issue of providing equal access to the platform for all students (see also subsection 5.7.1). This emerged because the features restrained to a non-identified user were considered key functionalities of the system. To supplant this issue, the creation of a web platform was suggested with the same functionalities of the smartphone. This would resolve entirely the problem because all the students have access to computers with internet on the school (for example in the library) and these devices are not seen as public devices (the probability of the student forgetting to log out is lower). 
Besides this issue, the teachers were pleased with the conceptual system that was built with their contribution and envisioned a great acceptance by the students.

\section{Conclusions and future work}

The work present in this paper suggest that digital public displays might have an important role in enticing learners to explore themes and share learning content within schools' public spaces. The teachers we engaged with were able to envision some of its potential. The workshops run were useful to generate ideas and make the design team aware of the specific challenges related to the school's context. Furthermore, the workshops were also able to create a sense of partnership between teachers and the design team towards the design and development. We believe this partnership will be crucial to foster wider adoption by the schools' communities.

In relation to the questions initially posed, the discussions in the workshops and the data collected seem to suggest that the teachers became aware of the digital public displays potential within the school's context. The interactive features referred to above were thoroughly discussed and made the design team aware of the constraints (the issue of allowing commenting the videos being displayed is a good example). The ethics issues raised need to be carefully taken into account in future iteration of the design. We will need to think of a design framework that will allow us to explore such theme.

Based on the design ideas already in place, our next step involves the deployment of a functional prototype in the schools. We will be able to collect data about the adoption and use of the different features included in the prototype (logs of the system, questionnaires and interviews). The data collected in conjunction with further design workshops with different groups of relevant users (teachers, students, and other potential stakeholders) will lead to further iterations of the design.

\section{ACKNOWLEDGMENTS}

The research leading to these results has received funding from the European Community's Seventh Framework Programme (FP7/2007-2013) under grant agreement no. 317964 JUXTALEARN.

We would like to sincerely thank all the teachers from Teknikum, Katedralskolan, Kronoberg Skola (Sweden) and Escola Secundária de Alberto Sampaio (Portugal), that agreed to participate in the design workshops.

\section{REFERENCES}

[1] Barradell, S. (2013). The identification of threshold concepts: a review of theoretical complexities and methodological challenges, Higher Education, 65 (2), pp. 265-276.

[2] Goodyear, P. (2011). Emerging methodological challenges for educational research. In L. Markauskaite, P. Freebody \& J. Irwin (Eds.), Methodological Choice and Design: Scholarship, Policy and Practice in Social and Educational Research (pp. 253-266). New York: Springer.

[3] Buckingham, D. \& Willett, R. (2006). Digital generations: Children, Young People, and New Media. Mahwah. New Jersey: Lawrence Erlbaum Associates.

[4] Coiro, J., Knobel, M., Lankshear, C. and Leu, D. (eds). (2008). The Handbook of Research on New Literacies. Mahwah. New Jersey: Lawrence Erlbaum Associates.

[5] Snyder, K; Panichi, L. \& Lindberg, O. (2010). On the issue of quality of experience in technology supported learning. Designs for Learning. No 1-2/ December 2010, pp. 42-51.

[6] Brignull, H. et al. (2004). The introduction of a shared interactive surface into a communal space. In Proceedings of the 2004 ACM conference on Computer supported cooperative work, pp.49-58.

[7] Jose, R.; Otero, N.; Izadi, S. \& Harper, R. (2008). Instant Places: Using Bluetooth for Situated Interaction in Public Displays.Pervasive Computing, IEEE 7 (4) (December), pp. 52-57.

[8] Otero, N.; José, R. \& Silva, B. (2012). Interactive Public Digital Displays: Investigating Its Use in a High School Context. In Proceedings of the Workshop on SOcial and MObile COmputing for Collaborative Environments (SOMOCO). LNCS 7567, p. 617.

[9] Agamanolis, S. (2003). Designing Displays for Human Connectedness. In K. O'Hara et al. (eds). Public and Situated Displays. Springer Netherlands, pp. 309-334.

[10] Huang, E.; Mynatt, E.; Russel, R. \& Sue, A. (2006). Secrets to Success and Fatal Flaws: The Design of Large-Display Groupware. IEEE Computer Graphics and Applications, 26 (1), pp: 37-45. 